\title{
Evaluation of Data Quality and Drought Monitoring Capability of FY-3A MERSI Data
}

\author{
Daxiang Xiang, ${ }^{1}$ Liangming Liu, ${ }^{1}$ Qiao Wang, ${ }^{2}$ Na Yang, ${ }^{1}$ and Tao Han ${ }^{3}$ \\ ${ }^{1}$ School of Remote Sensing and Information Engineering, Wuhan University, Wuhan Hubei 430079, China \\ ${ }^{2}$ Network \& Information Center, Changjiang Water Resources Commission, Wuhan Hubei 430010, China \\ ${ }^{3}$ Key Laboratory of Arid Climatic Change and Reducing Disaster of Gansu Province and KeyOpen Laboratory of Arid Climatic Change \\ and Reducing Disaster of CMA, Institute of Arid Meteorology, Lanzhou Gansu 730020, China
}

Correspondence should be addressed to Daxiang Xiang, daxiangx@163.com

Received 22 September 2009; Accepted 18 November 2009

Academic Editor: Djamel Bouchaffra

Copyright () 2010 Daxiang Xiang et al. This is an open access article distributed under the Creative Commons Attribution License, which permits unrestricted use, distribution, and reproduction in any medium, provided the original work is properly cited.

\begin{abstract}
FY-3A is the second Chinese Polar Orbital Meteorological Satellite with global, three-dimensional, quantitative, and multispectral capabilities. Its missions include monitoring global disasters and environment changes. This study describes some basic parameters and major technical indicators of the FY-3A and evaluates data quality and drought monitoring capability of the Medium-Resolution Imager (MERSI) onboard the FY-3A. Data obtained with the MERSI was compared with that of the MODerate-resolution Imaging Spectroradiometer (MODIS), imaged at the same time period and geographic zone. In addition, the Temperature/Vegetation Drought Index (TVDI), a highly accurate and stable monitoring model, was used to monitor drought condition with MERSI and MODIS sensors. It is found in the study that the relative accuracy of data, obtained with these two devices, was consistent with the acceptable overall accuracy of 93.8. Furthermore, spatial resolution of MERSI is superior as compared to that of MODIS. Therefore, FY-3A MERSI can serve a reliable and new data source for drought monitoring.
\end{abstract}

\section{Introduction}

1.1. Drought Monitoring. Drought is a normal, recurrent feature of climate. It occurs almost everywhere, although its features vary from region to region. In the most general sense, drought originates from a deficiency of precipitation over an extended period of time, resulting in a water shortage for some activity, group, or environmental sector (NDMC define). Consequently, it is required to demonstrate the distribution and degree of drought condition timely, which is crucial for drought warning and resisting effectively [1-3].

At present, remote sensing methods for drought monitoring are mainly classified into four categories: Vegetation Index-based $[4,5]$, Temperature-based [6], Vegetation and Temperature-based [7], and Cloud-based [8]. The representative indicies include Vegetation Supply Water Index (VSWI) [9], Temperature/Vegetation Dryness Index (TVDI) $[10,11]$, Apparent Thermal Inertia Index (ATI) $[12,13]$, and Cloud Parameters Index (CPI) [14]. As FY-3A satellite is a polar-orbital, its temporal resolution is hard to meet the requirements of cloud-based method, so CPI is not utilized in this study. ATI requires the index of temperature variation between day and night. However, polar-orbital satellites cannot provide real-time temperature; consequently ATI method is not suitable for FY-3A to monitor drought. VSWI and TVDI need only two parameters-Normal Difference Vegetation Index (NDVI) and Land Surface Temperature (LST), which can be calculated from MERSI data. Meanwhile, taking the superiority of TVDI into account, it is be used in this study.

1.2. FY-3A MERSI. The FY-3A satellite is the second Chinese polar orbital meteorological satellite which provides global, three-dimensional, high-quantified, and multispectral remote sensed data. The satellite weighs $2400 \mathrm{~kg}$, and has in-orbit dimensions of $4.46 \times 10 \times 3.79$ meters. Its orbit altitude is $836.4 \mathrm{~km}$, with an obliquity of 98.753 degrees and an orbit time of 101.496 minutes. Its designed life-span is over 2 years. FY-3A has refined the sensor technology, which is multisensor, microwave, hundred-meter resolution, and global data reception rather than single-sensor, optical 


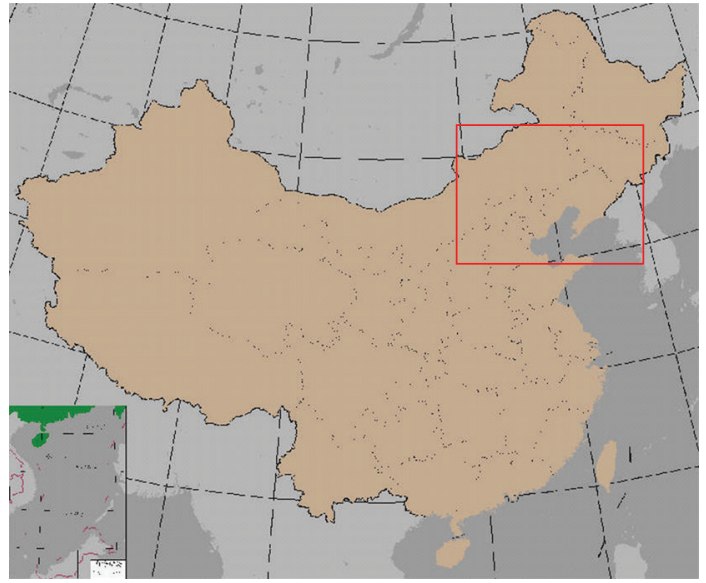

FIgure 1: Study area in North China.

only, kilo-meter resolution, and domestic data reception in the field of meteorological satellite. FY-3A missions include monitoring natural disasters and environmental changes all over the world and offering meteorological information for agriculture. Since, drought monitoring has naturally become one of the important applications of FY-3A. Therefore, drought monitoring capability of FY-3A deserves a comprehensive evaluation. Using MODIS results obtained at the same time period as reference, data quality, and drought monitoring capability of MERSI is analyzed in the study (CMA).

With multispectral and high-resolution features, MERSI can detect atmosphere, land, and ocean through the reflection of visible channels and thermal infrared radiation. There are three channels located in the water vapor absorption window $(0.905 \sim 0.980 \mathrm{um})$ which increase detection capability for atmospheric water vapor, in particular water vapor at low levels. Visible bands with spatial resolution of 250 meters can be utilized to derive NDVI and thermal bands for LST. Hence, MERSI has an enormous potential for drought monitoring. Details about some spectral properties of MERSI are listed in Table 1.

It can be found in Table 1 that FY-3A MERSI has red, near-infrared, and thermal infrared channels with spatial resolution of 250 meters, so it can provide drought monitoring information of 250 meters. This should be a great improvement of meteorological satellite data in spatial resolution for drought monitoring application.

Both FY-3A and TERRA are polar-orbiting satellites. Channels setting of MERSI, including the center wavelength and the wave width, is basically consistent with that of MODIS, in particular the bands used for monitoring surface vegetation, atmospheric water vapor, and surface temperature. MODIS data is calibrated on orbit and it uses the complicated recorrecting technology to locate when it scans. Because of high-quality and effective monitoring, MODIS has become a widely used data source in drought monitoring. Therefore, MODIS is optimal reference data in analyzing and evaluating radiometric calibration, relative geometric location, and drought monitoring results of MERSI.

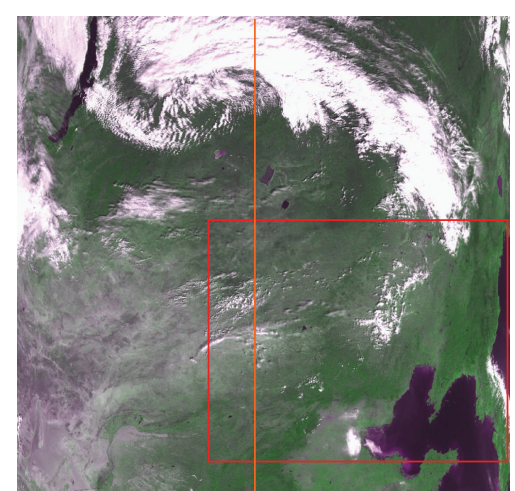

(a)

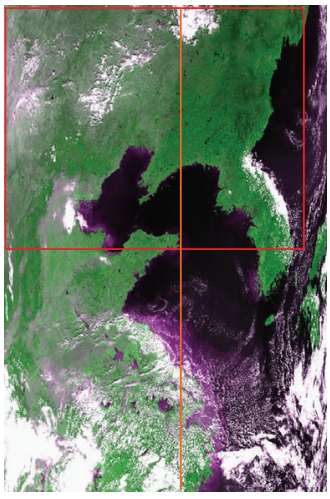

(b)

FIgure 2: Original data (a) FY-3A MERSI, and (b) TERRA MODIS.

In order to enlarge the remote sensing data source, the objective of the present study is to evaluate the data quality and the drought monitoring capability of FY-3A MERSI data with TERRA MODIS data as a reference.

\section{Materials and Methods}

2.1. Study Area. The case study area is near Bohai Bay in North China (area inside the red frame, Figure 1) with latitude range from $37^{\circ} \mathrm{N}$ to $46^{\circ} \mathrm{N}$ and longitude range from $109^{\circ} \mathrm{E}$ to $129^{\circ} \mathrm{E}$, respectively. There are a variety of land covers in the area, including vegetation, bare area, and waters.

2.2. MERSI and MODIS Data. The data of the study were extracted from MERSI on 1st October 2008, at 03:00 AM (GMT) (Figure 2(a)), and from TERRA-MODIS of the same area 15 minutes earlier (Figure 2(b)). The image of MERSI was $2000 \times 2084$ pixels, of TERRA-MODIS $1354 \times 2030$ pixels. To make it comparable with the result of MODIS data, FY-3A MERSI data was resampled to 1000-meter spatial resolution. These two kinds of data were provided by China Meteorological Administration (CMA) and MODIS Data Receiving Station in Wuhan University, respectively.

The orange lines in Figure 2 denote orbit central lines. Their spatial resolution has achieved the anticipated design requirement and the texture information is plentiful. The 
TABLE 1: Spectral properties of MERSI (partial).

\begin{tabular}{lccccc}
\hline Channel & Wavelength $(\mathrm{um})$ & Bandwidth $(\mathrm{um})$ & Resolution $(\mathrm{m})$ & Ne $\Delta \rho(\%)$ & Application \\
\hline 3 & 0.650 & 0.05 & 250 & 0.4 & Vegetation monitoring \\
4 & 0.865 & 0.05 & 250 & 0.45 & Temperature retrieve \\
5 & 11.25 & 2.5 & 250 & $0.50 \mathrm{~K}$ & 0.10 \\
17 & 0.905 & 0.02 & 1000 & 0.10 & Atmospheric Water Vapor detection \\
18 & 0.940 & 0.02 & 1000 & 0.10 & \\
\hline
\end{tabular}

study area is in the lower right corner of MERSI data (Figure 2(a)) and at the upper left side of MODIS data (Figure 2(b)).

\subsection{Methods}

2.3.1. NDVI. The Normalized Difference Vegetation Index is a satellite-derived global vegetation indicator obtained from the red, near-infrared (NIR) ratio of vegetation reflectance in the electromagnetic spectrum $[15,16]$

$$
\mathrm{NDVI}=\frac{\rho_{\mathrm{IR}}-\rho_{\mathrm{R}}}{\rho_{\mathrm{IR}}+\rho_{\mathrm{R}}} .
$$

The parameters, $\rho_{\mathrm{IR}}$ and $\rho_{\mathrm{R}}$, are the reflectivity of nearinfrared and red channels, respectively.

NDVI provides information on vegetation productivity and phenology over large temporal and spatial scales and has been widely used in the recent ecological studies as a proxy for vegetation productivity and phenology [17].

2.3.2. LST. The Land Surface Temperature can be calculated from the Brightness Temperature (BT) of the thermal infrared channels based on the thermal transmission equation. Because FY-3A MERSI contains only one thermal infrared channel, LST is replaced by BT of the thermal infrared channel in this paper [18].

2.3.3. TVDI. Sandholt and others extracted water stress indices (i.e., the index of temperature vegetation drought) on the basis of the simplified NDVI-LST feature space. LST $_{\text {min }}$ (minimum LST) is a straight-line paralleling with NDVI axis, and $\mathrm{LST}_{\max }$ (maximum LST) has a linear relation with NDVI. The TVDI can be obtained with formula (2) on the basis of NDVI-LST feature space $[19,20]$ :

$$
\mathrm{TVDI}=\frac{\mathrm{LST}-\mathrm{LST}_{\text {min }}}{\mathrm{LST}_{\text {max }}-\mathrm{LST}_{\text {min }}} .
$$

In fact, $\mathrm{LST}_{\min }$ in the NDVI-LST feature space varies under different vegetation coverage conditions [21-23]. Formula (2) is obtained by calculating the four Vertex Coordinates of trapezium theoretically based on the assumption that NDVI-LST feature space is trapezoidal. Therefore, $\mathrm{LST}_{\min }$ and $\mathrm{LST}_{\max }$ can be obtained through Least-squares linear fitting when NDVI-LST feature space is simplified into a triangle. In traditional studies, the function between $\mathrm{LST}_{\min }$ and NDVI is defined as wet side equation while that of $\mathrm{LST}_{\max }$ is accordingly called dry side equation. The formulas are as follows:

$$
\begin{aligned}
& \mathrm{LST}_{\text {min }}=a_{1}+b_{1} \mathrm{NDVI}, \\
& \mathrm{LST}_{\text {max }}=a_{2}+b_{2} \mathrm{NDVI},
\end{aligned}
$$

where $a_{1}$ and $b_{1}$ are the coefficients of the dry side equation, and $a_{2}$ and $b_{2}$ are the coefficients of the wet side equation, respectively.

In the experiment, a serial points (e.g., take $\mathrm{LST}_{\min }$ ), $\left(\mathrm{NDVI}_{i}, \mathrm{LST}_{\min , i}\right)$ is extracted, where $i$ is the serial number of these point. Thus, the error matrix equation can be established

$$
V=\left[\begin{array}{c}
V_{1} \\
V_{2} \\
\vdots \\
V_{n}
\end{array}\right]=\left[\begin{array}{c}
1, \mathrm{NDVI}_{1} \\
1, \mathrm{NDVI}_{2} \\
\vdots \\
1, \mathrm{NDVI}_{n}
\end{array}\right]\left[\begin{array}{c}
a_{1} \\
b_{1}
\end{array}\right]-\left[\begin{array}{c}
\mathrm{LST}_{\mathrm{min}, 1} \\
\mathrm{LST}_{\mathrm{min}, 2} \\
\vdots \\
\mathrm{LST}_{\mathrm{min}, n}
\end{array}\right],
$$

where $V$ is the error matrix, and $n$ is the number of points.

The normal equation can be deduced from the error matrix equation based on the Least-squares algorithm

$$
\begin{aligned}
& {\left[\begin{array}{c}
1, \mathrm{NDVI}_{1} \\
1, \mathrm{NDVI}_{2} \\
\vdots \\
1, \mathrm{NDVI}_{n}
\end{array}\right]^{T}\left[\begin{array}{c}
1, \mathrm{NDVI}_{1} \\
1, \mathrm{NDVI}_{2} \\
\vdots \\
1, \mathrm{NDVI}_{n}
\end{array}\right]\left[\begin{array}{c}
a_{1} \\
b_{1}
\end{array}\right]} \\
& =\left[\begin{array}{c}
1, \mathrm{NDVI}_{1} \\
1, \mathrm{NDVI}_{2} \\
\vdots \\
1, \mathrm{NDVI}_{n}
\end{array}\right]^{T}\left[\begin{array}{c}
\mathrm{LST}_{\text {min }, 1} \\
\mathrm{LST}_{\text {min }, 2} \\
\vdots \\
\mathrm{LST}_{\text {min }, n}
\end{array}\right],
\end{aligned}
$$



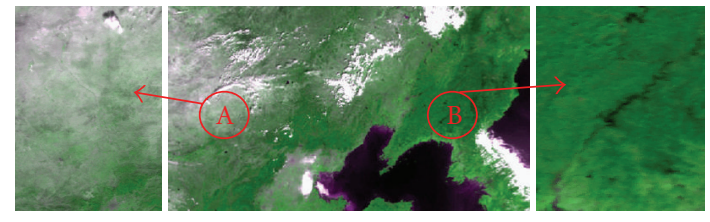

(a)
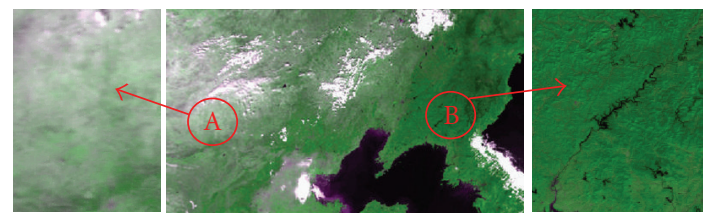

(b)

FIGURE 3: Result of radiation calibration and projection (true color: red, near-infrared, red): (a) FY-3A MERSI, and (b) TERRA MODIS.

The coefficients can be calculated based on the matrix operation:

$$
\begin{aligned}
{\left[\begin{array}{l}
a_{1} \\
b_{1}
\end{array}\right]=} & \left(\left[\begin{array}{c}
1, \mathrm{NDVI}_{1} \\
1, \mathrm{NDVI}_{2} \\
\vdots \\
1, \mathrm{NDVI}_{n}
\end{array}\right]^{T}\left[\begin{array}{c}
1, \mathrm{NDVI}_{1} \\
1, \mathrm{NDVI}_{2} \\
\vdots \\
1, \mathrm{NDVI}_{n}
\end{array}\right]\right) \\
& \times\left[\begin{array}{c}
1, \mathrm{NDVI}_{1} \\
1, \mathrm{NDVI}_{2} \\
\vdots \\
1, \mathrm{NDVI}_{n}
\end{array}\right]^{T}\left[\begin{array}{c}
\mathrm{LST}_{\min , 1} \\
\mathrm{LST}_{\min , 2} \\
\vdots \\
\mathrm{LST}_{\min , n}
\end{array}\right]
\end{aligned}
$$

where -1 is the inversion operation of matrix.

Last, the relation square ( $R$-square, $R^{2}$ ) demonstrates that this statistic measures how successful the fit is in explaining the variation of the data. A value closer to 1 indicates a better fit

$$
R^{2}=\frac{\left(\sum_{i=1}^{n}\left(\mathrm{LST}_{\min , i}-\overline{\mathrm{LST}_{\min }}\right)\left(\mathrm{NDVI}_{i}-\overline{\mathrm{NDVI}}\right)\right)^{2}}{\sum_{i=1}^{n}\left(\mathrm{LST}_{\min , i}-\overline{\mathrm{LST}_{\min }}\right) \cdot \sum_{i=1}^{\mathrm{n}}\left(\mathrm{NDVI}_{i}-\overline{\mathrm{NDVI}}\right)},
$$

where $\overline{\mathrm{LST}_{\text {min }}}$ is the average value of all $\mathrm{LST}_{\min }$, and $\overline{\mathrm{NDVI}}$ is the average of all NDVI.

\section{Result and Discussion}

After calibration, MERSI and MODIS data are projected with the latitude and longitude information recorded in scan. The relative accuracy of geometric location and radiometric calibration of the two kinds of data are also analyzed. NDVI is obtained through red and near infrared channels, and LST in the clear sky or cloud top temperature can be retrieved through thermal infrared and vapor channels. Furthermore, it is necessary to compare and analyze the distribution character of the dry and wet sides of the two images in the NDVI-LST feature space. User's accuracy, producer's accuracy, and the overall accuracy are evaluated for TVDI with MERSI and MODIS data.

\subsection{Data Preprocessing}

3.1.1. Calibration. The visible channels of MERIS are directly calibrated with slope-intercept form, and revised solar altitude and Earth-Sun Distance at the imaging moment. Digital number (DN) of infrared channels is already radiation value, so the brightness temperature can be gained with Planck Formula.

The visible data of MODIS is calibrated with slopeintercept form, while the infrared channel is calibration with Planck Formula to get brightness temperature directly [24-26]. The in-lab calibration accuracy of MERSI visible and infrared channels is $7 \%$ and $1 \mathrm{~K}$, respectively, but the radiation calibration accuracy of MODIS data is much higher.

Both record the latitude and longitude values in imaging; they are translated into geographic coordinates in the corresponding projection through strict transformation calculation method $[27,28]$. At the same time, gray value of a pixel is extracted to a corresponding location in the new image. The new projection is Lambert Equal-angle Intercross Taper Projection (Lambert Conformal Conic). Its specific parameters include Spheroid of Krasovsky, Datum of Krasovsky, the first standard parallel latitude of $47^{\circ} \mathrm{N}$, the second standard parallel latitude of $25^{\circ} \mathrm{N}$, the central meridian latitude of $36^{\circ} \mathrm{N}$, the central meridian longitude of $105^{\circ} \mathrm{E}$ and the origin coordinates of (5000000.0, 5000000.0) (measured in meters). The results of radiometric calibration and projection of the two data are shown in Figure 3.

It is obvious in Figure 3 that the two images have the similar color effect and the color of MERSI is slightly lighter than that of MODIS. Moreover, there is small difference in atmospheric conditions, for example, the sizes of cloud cluster are a little different at the Bohai Bay region. For region $B$ in Figures 3(a) and 3(b), it can be easy noticed that the resolution of MERSI data, located at the right side of original images, is coarser than that of MODIS which locates at the center of the original scene. Furthermore, the partial textures information of region B in MERSI data is poorer than that of MODIS. For region A in Figures 3(a) and 3(b), it is vice versa. This is mainly due to the different positions of the study area in the original images. Some statistic parameters can be seen in Table 2, and the gray histograms of selected channels are shown in Figure 4.

The population characteristic value different of red channel reflectivity between MERSI and MODIS data is 0.05 and the population variance is 0.02 . In other words, red channels of two sensors have same sensitivity to different types of ground objects. Of course, those asynchronous images and the differences of central wavelength and wave width will also have some effect on the scaling result, and this is beyond the scope of this study. The similar situation also happens to the reflectivity of near infrared channel. 
TABLE 2: Compared analysis of calibration result.

\begin{tabular}{|c|c|c|c|c|c|c|}
\hline \multirow{2}{*}{ Index } & \multicolumn{2}{|c|}{ Red channel } & \multicolumn{2}{|c|}{ Near-infrared channel } & \multicolumn{2}{|c|}{ Thermal infrared channel } \\
\hline & MODIS (b01) & MERSI (b03) & MODIS (b02) & MERSI (b04) & MODIS (b31) & MERSI (b05) \\
\hline Wavelength & 0.645 & 0.65 & 0.859 & 0.865 & 11.03 & 11.25 \\
\hline Mean & 0.141 & 0.192 & 0.238 & 0.303 & 290.76 & 290.55 \\
\hline Maximum & 1 & 1 & 1 & 1 & 304 & 301 \\
\hline Minimum & 0.02 & 0.05 & 0.01 & 0.02 & 226 & 234 \\
\hline Medium & 0.13 & 0.16 & 0.24 & 0.3 & 292 & 292 \\
\hline Std. Dev & 0.101 & 0.126 & 0.12 & 0.152 & 6.868 & 5.643 \\
\hline
\end{tabular}

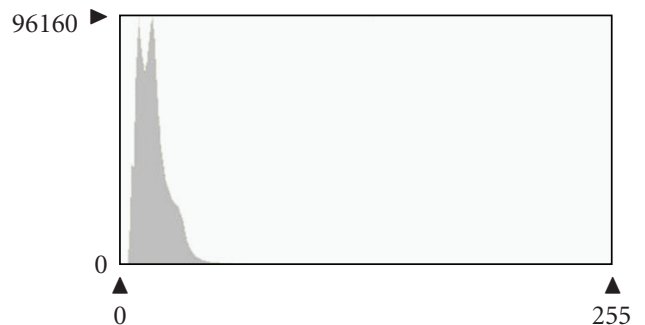

(a)

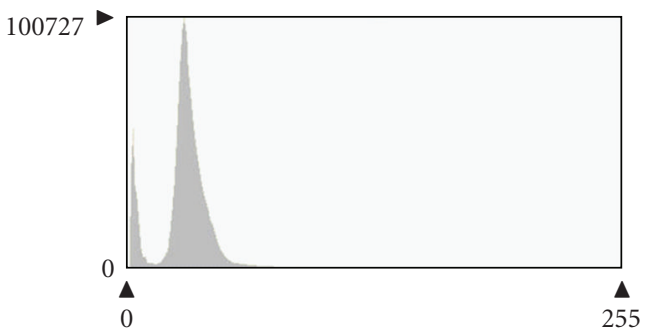

(c)

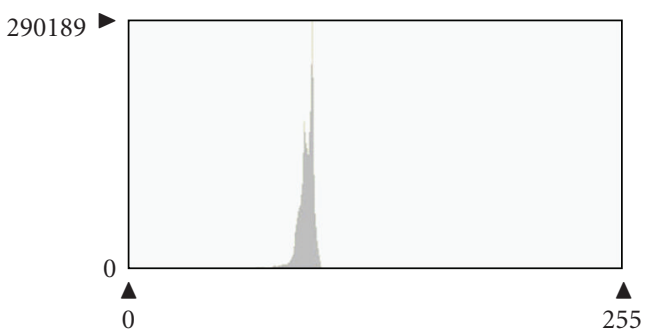

(e)

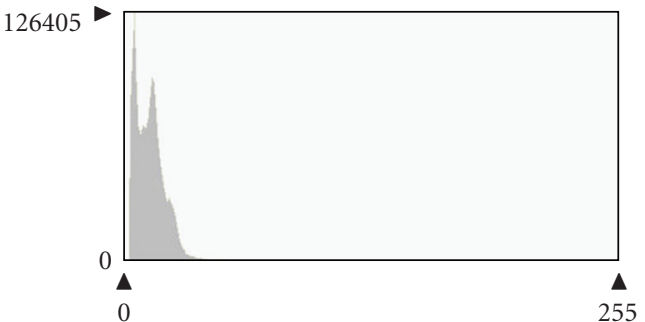

(b)

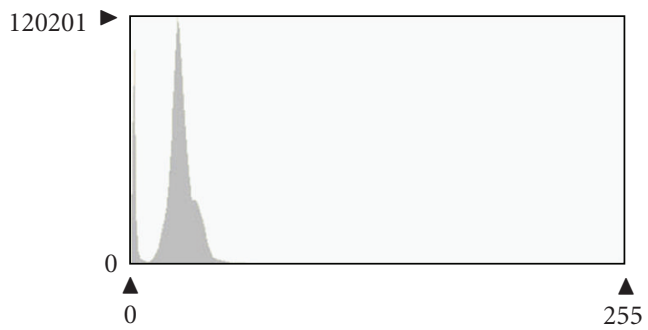

(d)

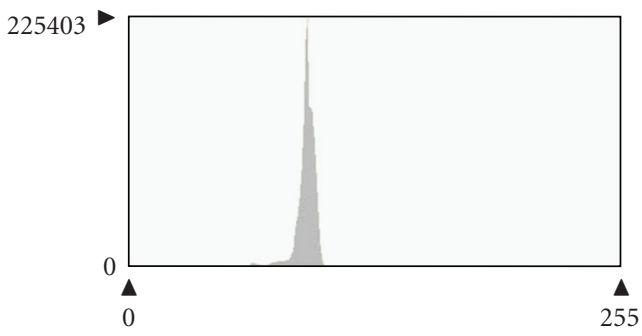

(f)

FIgURE 4: (a) MERSI red channel (reflectance $* 100$ ), (b) MODIS red channel (reflectance $* 100$ ), (c) MERSI near-infrared channel (reflectance $* 100$ ), (d) MODIS near-infrared channel (reflectance $* 100$ ), (e) MERSI thermal infrared channel (LST-200), and (f) MODIS thermal infrared channel (LST-200).

Its population characteristic value difference between the MERSI and MODIS data is 0.06 and the population variance is 0.02 . As can be seen from the reflectivity histogram of near infrared channel of MERSI and MODIS data, the histogram has two-peak values. According to the reflection spectral characteristics of ground objects, the lower peak value of reflectivity mainly results from the lower reflectivity of the surface waters, as most of the study area is covered by waters. Furthermore, LST in the clear sky or cloud top temperatures retrieved from two images are of little difference, and this indicates that MERSI has a strong capability for LST retrieving.
3.1.2. Relative Geometric Location Analysis. MODIS data is of high orbital location accuracy, so it can serve as reference for analyzing geometric location accuracy of MERSI data. Fifteen control points (balanced distributed in study area) are selected manually in the study. The geometric location differences of these points are used to analyze geometry location accuracy of MERSI data. Figure 5 shows that the spatial distribution of the points and Table 3 shows the geometric location difference of each point between two images.

It is shown in Table 3 that MERSI data has quite good location accuracy when compared with MODIS data. Its 
TABLE 3: Geometric location accuracy of MERSI data (unit: meter).

\begin{tabular}{|c|c|c|c|c|c|c|c|}
\hline Point no. & MODISX & MODISY & MERSIX & MERSIY & X_Diff & Y_Diff & RMS \\
\hline 1 & 5645738 & 5524095 & 5645718 & 5524098 & -682 & 186 & 707.32 \\
\hline 2 & 5932799 & 5861170 & 5932795 & 5862104 & -552 & -317 & 637.26 \\
\hline 3 & 6491754 & 5291080 & 6491693 & 5292060 & -104 & -176 & 205.46 \\
\hline 4 & 6854745 & 5403135 & 6853753 & 5404095 & -68 & 121 & 139.76 \\
\hline 5 & 7000293 & 5764656 & 6998805 & 5765990 & -160 & 128 & 205.96 \\
\hline 6 & 6636750 & 6044083 & 6635740 & 6045110 & -90 & 353 & 364.98 \\
\hline 7 & 6371813 & 5412078 & 6371707 & 5413082 & 241 & -173 & 297.46 \\
\hline 8 & 6129717 & 6003122 & 6128731 & 6003088 & 202 & 705 & 734.52 \\
\hline 9 & 5576752 & 6133008 & 5573799 & 6133098 & 233 & 15 & 233.74 \\
\hline 10 & 5417760 & 5347094 & 5415739 & 5347072 & 504 & -136 & 522.30 \\
\hline 11 & 6884743 & 6095120 & 6882747 & 6097091 & 67 & -381 & 387.63 \\
\hline 12 & 6299718 & 5735132 & 6298756 & 5736088 & 986 & 11 & 986.91 \\
\hline 13 & 6338741 & 6141127 & 6337732 & 6142106 & -143 & 137 & 198.29 \\
\hline 14 & 5409658 & 5642143 & 5406747 & 5642105 & 612 & -46 & 614.34 \\
\hline 15 & 5798758 & 5283109 & 5798707 & 5283129 & -229 & 232 & 326.44 \\
\hline Total difference & & & 0335 & 1443 & 466.5654 & & \\
\hline
\end{tabular}

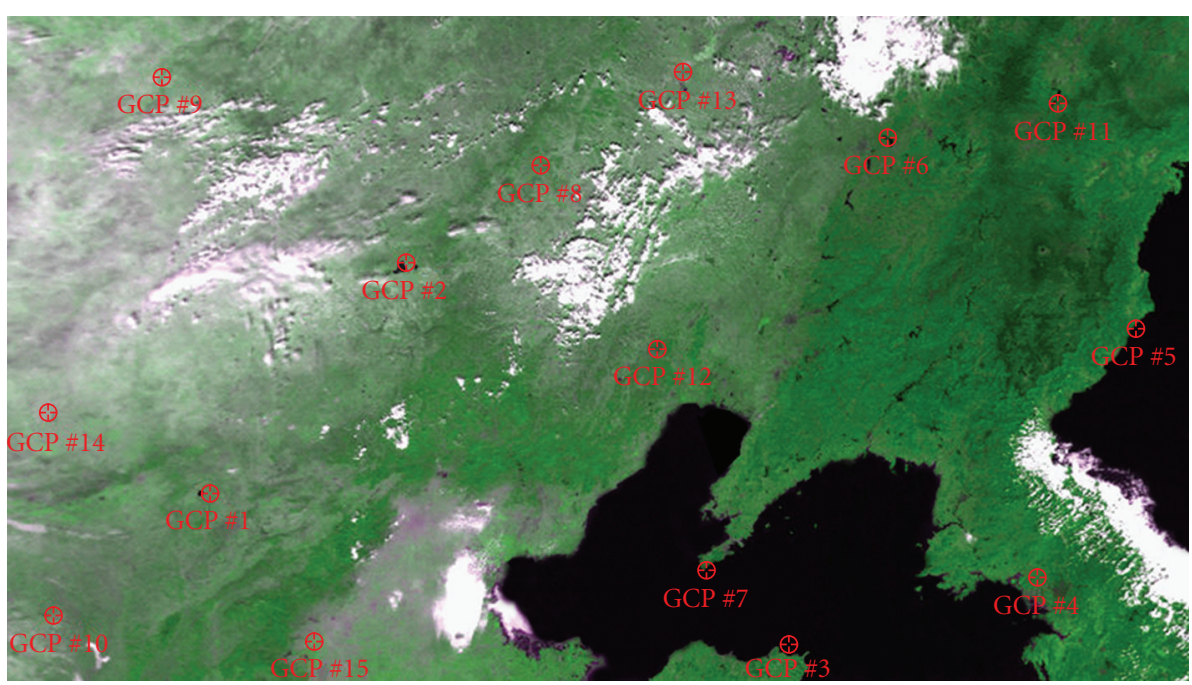

FIgURE 5: The distribution of the matching points.

overall difference is 0.4666 pixels, with $X$ direction difference of 0.415 pixels and $Y$ direction difference of 0.2131 pixels. The maximum difference point for $X$ is the point no. 12 with a small difference of $Y$, mainly because of manual pointchoosing. Actual spatial resolution of the points at the side is coarser than those near nadir, so the points at the left side of MODIS (no. 1, no. 2, no. 8, no. 12, and no. 14) have poor matching with their corresponding points near nadir of MERSI.

Based on the analysis of calibration results, MERSI can retrieve similar reflectivity, LST, and cloud top temperature as MODIS does. The geometric location difference between MERSI and MODIS is less than half pixel, while difference of some areas reaches one pixel, but it is still within the tolerance. In other words, the geolocation of MERSI is acceptable. Therefore, the MERSI data has potential for drought monitoring on basis of calibration and relative geometric location analysis.

3.2. Cloud Detection. NDVI and LST, the two key parameters for TVDI method, cannot be retrieved when remote sensing data is covered by clouds, so TVDI is inapplicable for the cloud-covered areas. Consequently, to identify whether an image is qualified for drought monitoring, cloud detection should be performed before deducing parameters [29]. In addition, areas covered by water need to be identified and ignored in case of error in establishing NDVI-LST feature space.

Cloud in visible band shows high reflectance, and remote sensing image with $0.66 \mathrm{um}$ is ideal for distinguishing borders between land and cloud [30]. The spectrum feature of cloud in NIR band (near $0.936 \mathrm{um}$ ) is mainly relevant 


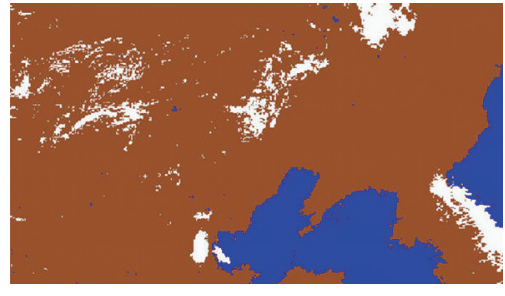

(a)

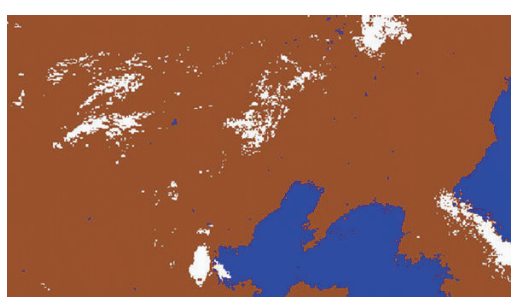

(b)

FIgURE 6: Result of cloud detection: (a) FY-3A MERSI, and (b) TERRA MODIS (brown is the clear sky, blue is water, white is cloud).

to water vapor content in atmosphere, so it can help to indicate the moisture feature, namely, absorption window. Because $0.66 \mathrm{um}$ and $0.936 \mathrm{um}$ clouds form sharp contrast with spectrum features of all kinds of ground objects, cloud information can be obtained by normalization, which not only gives prominence to cloud information and partially eliminate ill effects caused by solar altitude, satellite sweep angle and atmosphere route radiation. Normalized Cloud Detection Index (CDI) [12, 21] is expressed as

$$
\mathrm{CDI}=\frac{\rho_{0.66 \mu \mathrm{m}}-\rho_{0.936 \mu \mathrm{m}}}{\rho_{0.66 \mu \mathrm{m}}+\rho_{0.936 \mu \mathrm{m}}} .
$$

As cloud refection spectrum has reflection characteristics at $0.66 \mathrm{um}$ and its absorption window at $0.936 \mathrm{um}$ is influenced by water vapor, the CDI is positive (CDI $>0$ ), because soil reflection spectra has little difference in reflection properties between $0.66 \mathrm{um}$ and $0.936 \mathrm{um}$, the CDI is close to 0 ; vegetation reflection spectra has low reflection at $0.66 \mathrm{um}$ and high reflection at $0.936 \mathrm{um}$, the CDI is negative (CDI $<0)$.

As the reflectance of water is higher than vegetation near the red band and the reflectance of vegetation is obviously higher than water round the NIR band (0.841 um-0.876 um) $[31,32]$, a typical bimodal distribution appears in the histogram in Figures 4(c) and 4(d). Thus, NDVI is adopted to enhance land-water contrast.

Based on the cloud detection and water extraction algorithms discussed above, it can be concluded that MERSI and MODIS data can have cloud and water detection with the same algorithm and the results is shown in Figure 6.

Figure 6 shows that the results of MERSI and MODIS are unanimous in most areas. However, as a result of difference in imaging time, there is a slight difference in the cloud detection at the top left corner of images due to small atmospheric condition change.
3.3. Extraction of Drought Feature Parameters. For MERSI and MODIS, NDVI is usually calculated by red band and NIR band. Meanwhile, MERSI has only one TIR band (10 $12.5 \mathrm{um}$ with $250 \mathrm{~m}$ resolution in nadir point), which can be used to extract LST in the clear sky or cloud top temperature. Qin Zhihao deduced a simple, feasible LST algorithm with high accuracy according to the equation of land surface heat conduction $[33,34]$ and this method can be quoted to process MERSI data.

From previous studies, it is known that the distribution of the wet and dry sides is significant in the two-dimensional feature space composed by NDVI and LST from MERSI. It is necessary to analyze the characteristics of the NDVI-LST feature space and determine the wet and dry sides.

LST is calculated with the NDVI datasets and singlewindow algorithm, $\mathrm{LST}_{\max }, \mathrm{LST}_{\min }$, of different NDVI conditions is extracted on the basis of 0.01 NDVI step (NDVI is among 0.3 to 0.7 for medium vegetation coverage), and then NDVI-LST feature space is obtained (Figure 7).

It is shown in Figure 7 that $\mathrm{LST}_{\max }$ and $\mathrm{LST}_{\min }$ have better correlation with NDVI in MERSI than in MODIS, but their linear correlations are quite consistent. This may be due to the difference of cloud detection, that is to say, the cloud top temperature may be considered as the minimum LST in the clear sky in the process of selecting MODIS LST samples. From Figure 7, MODIS has smaller LST $_{\text {min }}$ and higher $\mathrm{LST}_{\max }$ for the same NDVI value. This demonstrates that MODIS data is doing better in retrieving LST in the clear sky than MERSI. Based on the principle of least squares solver fitting, the relationship functions are regressed in the NDVI-LST feature space. Because wet and dry side fitting of the low and high vegetation coverage (with NDVI ranging from 0 to 0.30 and from 0.70 to 1 , resp.) is quite instable, only middle NDVI of 0.30 to 0.70 is used in regression.

The fitting equations of wet and dry sides based on MERSI are as follows.

Dry side equation:

$$
\mathrm{LST}_{\max }=34.550-17.90 \mathrm{NDVI}, \quad R^{2}=0.8307 .
$$

Wet side equation:

$$
\mathrm{LST}_{\min }=-1.643+28.47 \mathrm{NDVI}, \quad R^{2}=0.9541 .
$$

The above formulas demonstrate that if the slope of dry side is less than zero, LST $_{\max }$ will decrease with the increase of vegetation coverage. Oppositely, the minimum LST is vice versa.

The fitting $R$-squares of MERSI wet and dry sides are 0.8307 and 0.9541 , respectively. It shows that changes of $\mathrm{LST}_{\text {max }}$ and $\mathrm{LST}_{\text {min }}$ are quite consistent with the changes of NDVI, and the mechanism of drought is well demonstrated. The fitting $R$-squares of MODIS are 0.6852 and 0.6904, respectively.

3.4. Drought Monitoring. It is found through the above analysis that the wet and dry sides possess high-fitting relevance in the NDVI-LST feature space of MERSI and it means that MERSI is suitable for drought monitoring. TVDI 
TABLE 4: TVDI confusion matrices between MERSI and MODIS.

\begin{tabular}{|c|c|c|c|c|c|c|c|}
\hline \multirow{2}{*}{ TERRA } & \multicolumn{7}{|c|}{ FY-3A } \\
\hline & High & Middle & Low & Normal & Wet & Cloud & Producer accuracy \\
\hline High & 0.1281 & 0.1949 & 0.056 & 0.0287 & 0.1276 & 0.097 & 20.235 \\
\hline Middle & 0.105 & 8.0814 & 7.7763 & 0.4183 & 0.3291 & 0.602 & 46.6786 \\
\hline Low & 0.1833 & 1.3909 & 18.945 & 4.3751 & 1.1041 & 0.533 & 71.4042 \\
\hline Normal & 0.110 & 0.1072 & 2.3245 & 7.5212 & 2.4723 & 0.239 & 58.8742 \\
\hline Wet & 0.2405 & 0.0579 & 0.3669 & 2.3293 & 21.2561 & 0.266 & 86.6994 \\
\hline Cloud & 0.204 & 0.4602 & 0.5915 & 0.31 & 0.4686 & 16.195 & 88.8404 \\
\hline User accuracy & 13.1908 & 78.517 & 63.024 & 50.199 & 82.523 & 90.296 & $\begin{array}{c}\text { Correct: } 72.1274 \\
\text { Acceptable: } 93.8306\end{array}$ \\
\hline
\end{tabular}

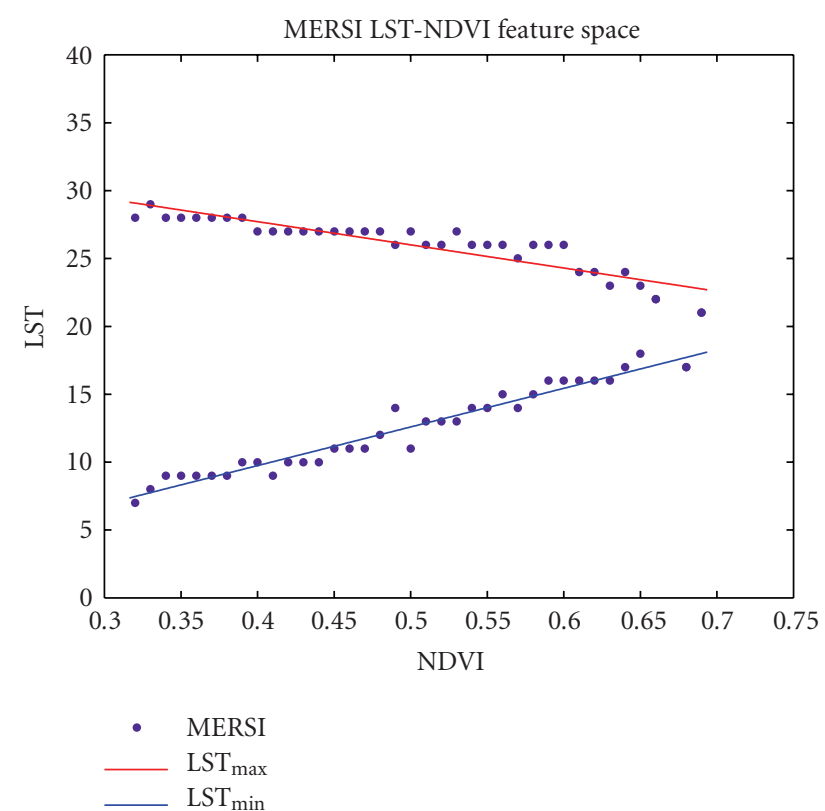

(a)

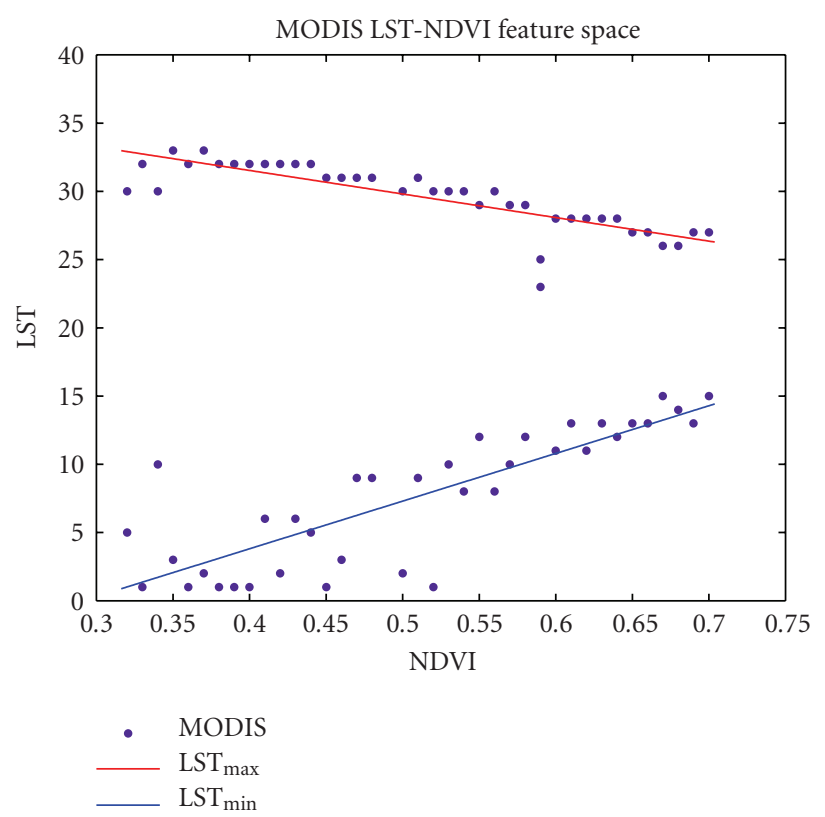

(b)

FIgURE 7: The wet and dry sides in feature space: (a) FY-3A MERSI, and (b) TERRA MODIS.

is used to analyze the drought monitoring capability of FY3A MERSI in the study. MODIS has been proved to be one of the most successful data for drought monitoring and is naturally utilized as reference data. The monitoring results and comparison of MERSI and MODIS are as shown in Figure 8.

Table 4 is comparison of TVDI confusion matrices between FY-3A MERSI and TERRA MODIS. User's accuracy, producer's accuracy, and overall accuracy of MERSI are analyzed with MODIS TVDI as a reference. The overall accuracy contains the correct and acceptable accuracy, where the correct accuracy indicates that the monitoring results of MERSI TVDI and MODIS TVDI are unanimous. The definition of the acceptable accuracy is that the difference of grade results is lower than one level. For instance, it is acceptable if one pixel is shown as severe drought in MERSI TVDI and its corresponding point shown as medium drought in MODIS TVDI. It is clear in Table 4 that MERSI has almost the same drought monitoring result with MODIS.
In other words, absolutely correct accuracy is 72.1274 and the acceptable accuracy is as high as 93.8306. Thus, MERSI is as strong as MODIS in drought monitoring.

\section{Conclusion}

The following conclusions can be reached on the basis of above comparison and analysis.

(1) FY-3A MERSI data enjoys a high quality. The relative difference of calibration results between MERSI and MODIS is negligible, and temperature retrieving capability of MERSI is as good as MODIS. In addition, it is also proved that their cloud detection results have strong consistency. Furthermore, the overall difference of geo-location between MERSI and MODIS is about 0.4666 pixels, in other words, MERSI data possess wonderful geo-location capability. 


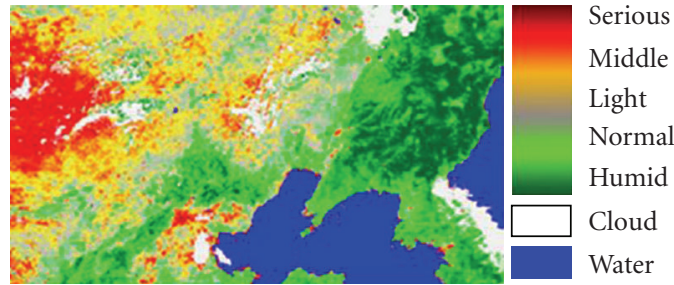

(a)

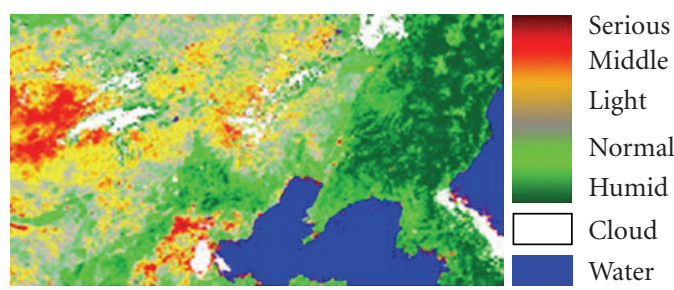

(b)

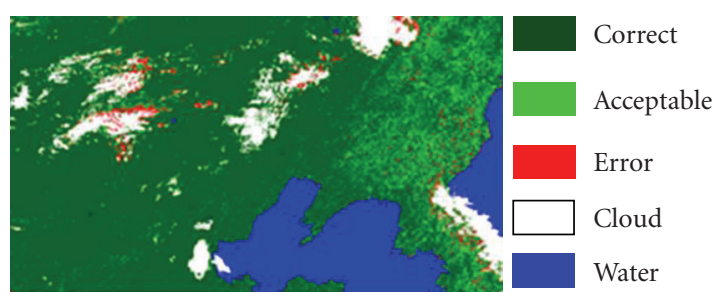

(c)

Figure 8: (a) TVDI from FY-3A MERSI, (b) TVDI from MODIS TERRA, and (c) TVDI Difference between MERSI and MODIS.

(2) The LST $T_{\max }$ decreases with the increase of vegetation coverage, vice versa for $\mathrm{LST}_{\min }$. The fitting R-squares of wet and dry sides extracted from MERSI are as high as 0.8307 and 0.9541 , respectively. It means that changes of $\mathrm{LST}_{\max }$ and $\mathrm{LST}_{\min }$ have high consistency with those of NDVI, and the mechanism of drought is well presented. While, the fitting $R$-squares of MODIS data (for wet and dry side) are, respectively, 0.6852 and 0.6904 which are lower than that of MERSI. From the NDVI-LST feature space of MERSI, the scatter points' distribution of wet side is highly correlated with that of dry side, and this is a clear demonstration of drought information.

(3) The TVDI confusion matrices indicate that FY-3A MERSI possesses the similar capability to MODIS, with absolute accuracy of 72.1274 and acceptable accuracy of 93.8306 . Therefore, it is possible for us to obtain the drought products in operation with $250 \mathrm{~m}$ spatial resolution and global scale with MERSI as a new kind of data.

\section{Acknowledgments}

The authors are grateful to the members of the MODIS Data Receiving Station, Wuhan University, China. This paper is granted by FY-3A Satellite Application in Drought Monitoring and Early Warning in Northwest China which is subtask of FY-3A Satellite Application Projection from China Meteorological Administration (CMA). Meanwhile, this paper is also granted the AMD University Plan.

\section{References}

[1] D. A. Wilhite and M. H. Glantz, "Understanding the drought phenomenon: the role of definitions," Water International, vol. 10, no. 3, pp. 111-120, 1985.

[2] R. Nemani, L. Pierce, S. Running, and S. Goward, "Developing satellite-derived estimates of surface moisture status," Journal of Applied Meteorology, vol. 32, no. 3, pp. 548-557, 1993.

[3] A. Volcani, A. Karnieli, and T. Svoray, "The use of remote sensing and GIS for spatio-temporal analysis of the physiological state of a semi-arid forest with respect to drought years," Forest Ecology and Management, vol. 215, no. 1-3, pp. 239-250, 2005.

[4] T. Tadesse, J. F. Brown, and M. J. Hayes, "A new approach for predicting drought-related vegetation stress: integrating satellite, climate, and biophysical data over the U.S. central plains," ISPRS Journal of Photogrammetry and Remote Sensing, vol. 59, no. 4, pp. 244-253, 2005.

[5] P. R. Bajgiran, A. A. Darvishsefat, A. Khalili, and M. F. Makhdoum, "Using AVHRR-based vegetation indices for drought monitoring in the Northwest of Iran," Journal of Arid Environments, vol. 72, no. 6, pp. 1086-1096, 2008.

[6] A. T. Jeyaseelan and F. N. Kogan, "Evaluation of GVI based indices for drought early warning in India," in Disaster Forewarning Diagnostic Methods and Management, vol. 6412 of Proceedings of SPIE, pp. 4120-4120, 2006.

[7] G. Marshall and X. Zhou, "Drought detection in semiarid regions using remote sensing of vegetation indices and drought indices," in Proceedings of the International Geoscience and Remote Sensing Symposium (IGARSS '04), vol. 3, pp. 15551558, September 2004.

[8] L. Liu, D. Xiang, X. Dong, and Z. Zhou, "Improvement of the drought monitoring model based on the cloud parameters method and remote sensing data," in Proceedings of the 1st International Workshop on Knowledge Discovery and Data Mining (WKDD '08), pp. 293-296, 2008.

[9] W. Mo, Z. Wang, H. Sun, L. Ma, and L. He, "Remote Sensing Monitoring of Farmland Drought Based on Vegetation Supply Water Index," Journal of Nanjing Institute of Meteorology, vol. 29, no. 3, pp. 396-401, 2006.

[10] C. Naira, L. Robert, M. Ramata, and T. Marouane, "Surface soil moisture status over the Mackenzie River Basin using a temperature/vegetation index," in Proceedings of the International Geoscience and Remote Sensing Symposium (IGARSS '07), pp. 1846-1848, 2007.

[11] N. R. Patel, R. Anapashsha, S. Kumar, S. K. Saha, and V. K. Dadhwal, "Assessing potential of MODIS derived temperature/vegetation condition index (TVDI) to infer soil moisture status," International Journal of Remote Sensing, vol. 30, no. 1, pp. 23-39, 2008.

[12] W. Li, S. Y. Mao, and W. Chen, "A new method of cloud detection in MODIS image," in Proceedings of the 7th International Conference on Electronic Measurement and Instruments, vol. 7, pp. 281-285, 2005.

[13] G. Cai, MODIS data based thermal inertia and land surface temperature modeling and their applications in determination of soil moisture and heat exchange, doctoral dissertation, M.S. thesis, Institute of remote sensing applications Chinese academy of sciences, 2006. 
[14] L. Liu, D. Xiang, Z. Zhou, and X. Dong, "Analyses the modification functions of the drought monitoring model based on the cloud parameters method," in Proceedings of the 1st International Congress on Image and Signal Processing (CISP' '08), vol. 4, pp. 687-691, 2008.

[15] B. C. Reed, J. F. Brown, D. VanderZee, T. R. Loveland, J. W. Merchant, and D. O. Ohlen, "Measuring phenological variability from satellite imagery," Journal of Vegetation Science, vol. 5, no. 5, pp. 703-714, 1994.

[16] S. Hamel, M. Garel, M. Festa-Bianchet, J.-M. Gaillard, and S. D. Côté, "Spring Normalized Difference Vegetation Index (NDVI) predicts annual variation in timing of peak faecal crude protein in mountain ungulates," Journal of Applied Ecology, vol. 46, no. 3, pp. 582-589, 2009.

[17] J. T. Kerr and M. Ostrovsky, "From space to species: ecological applications for remote sensing," Trends in Ecology \& Evolution, vol. 18, no. 6, pp. 299-305, 2003.

[18] Y. Julien and J. A. Sobrino, "The Yearly Land Cover Dynamics (YLCD) method: an analysis of global vegetation from NDVI and LST parameters," Remote Sensing of Environment, vol. 113, no. 2, pp. 329-334, 2009.

[19] I. Sandholt, K. Rasmussen, and J. Andersen, "A simple interpretation of the surface temperature/vegetation index space for assessment of surface moisture status," Remote Sensing of Environment, vol. 79, no. 2-3, pp. 213-224, 2002.

[20] S. Qi, C. Wang, and Z. Niu, "Evaluating soil moisture states in China using the temperature/vegetation dryness index(TVDI)," Joutnal of Remote Sensing, vol. 15, pp. 420-427, 2003.

[21] W. Li and D. Li, "The universal cloud detection algorithm of MODIS data," in Geoinformatics 2006: Remotely Sensed Data and Information, vol. 6419 of Proceedings of SPIE, p. 4190, October 2006.

[22] C. Li and H. Li, "Study on winter wheat drought monitoring by TVDI in Hebei Province," in Remote Sensing and Modeling of Ecosystems for Sustainability III, vol. 6298 of Proceedings of SPIE, pp. 2981-2981, San Diego, Calif, USA, August 2006.

[23] C. Jin, Q. M. Qin, L. Zhu, P. Nan, and A. Ghulam, "TVDI based crop yield prediction model for stressed surfaces-a case study on Ningxia Huizu autonomous region of China," in Proceedings of the International Geoscience and Remote Sensing Symposium (IGARSS '08), pp. 4656-4658, 2008.

[24] J. Tong, K. Qiu, X. Li, and Z. Yang, "Radiometric crosscalibration of the FY1D/VIRR and EOS/MODIS in the visible and near-infrared spectral bands," Journal of Remote Sensing, vol. 4, pp. 349-356, 2005.

[25] G.-K. Zhang, C. Chen, F. Xing, H.-Y. Zhang, and Y.-S. Zhao, "Spectral radiometric calibration research of Quick Bird digital image," Spectroscopy and Spectral Analysis, vol. 28, no. 3, pp. 494-498, 2008.

[26] Z. Zhang and G. He, "Radiometric calibration of Landsat 5 TM data," Science and Technology Review, vol. 7, pp. 54-58, 2008.

[27] Z. M. Cheng, L. Lu, and X. M. Qiu, "Make use of the space projection method to carry on the geometric exact correction to the remote sensing picture," Science Mosaic, vol. 10, pp. 103$105,2005$.

[28] F. Duan, L. Zhu, and L. Gao, "Realization and optimization of multi-projection transformation on remote sensing image," Journal of Image and Graphics, vol. 5, pp. 608-610, 2005.

[29] D. C. Bamber, S. Mackin, and P. Palmer, "Cloud detection and height estimation through registration of disaster monitoring constellation imagery," in Remote Sensing of Clouds and the Atmosphere XI, vol. 6362 of Proceedings of SPIE, pp. 448-457, September 2006.
[30] T. Cheng, X. Gu, L. Chen, T. Yu, and G. Tian, "Cloud detection based on the spectral, multi-angular, and polarized characteristics of cloud," in Proceedings of the IEEE International Geoscience and Remote Sensing Symposium (IGARSS '08), pp. 3321-3324, Barcelona, Spain, June 2008.

[31] X. Wu and Q. Cheng, "Study on methods of cloud identification and data recovery for MODIS data," in Remote Sensing of Clouds and the Atmosphere XII, vol. 6745 of Proceedings of SPIE, pp. 7450-7450, Florence, France, September 2008.

[32] H. Wang, Y. He, and H. Guan, "Application of support vector machines in cloud detection using EOS/MODIS," in Remote Sensing Applications for Aviation Weather Hazard Detection and Decision Support, vol. 7088 of Proceedings of SPIE, p. 880, San Diego, Calif, USA, August 2008.

[33] Z.-H. Qin, M.-H. Zhang, A. Karnieli, and P. Berliner, "Monowindow algorithm for retrieving land surface temperature from Landsat TM6 data," Acta Geographica Sinica, vol. 56, no. 4, pp. 456-466, 2001.

[34] D. M. Song, N. K. Hu, Y. L. Wu, and X. Y. Li, “The study on the ground surface parameters in Minqin Oasis in Gansu Province with using remote sensing data," in Proceeding of the International Conference on Informational Technology and Environmental System Science, vol. 4, pp. 339-343, 2008. 

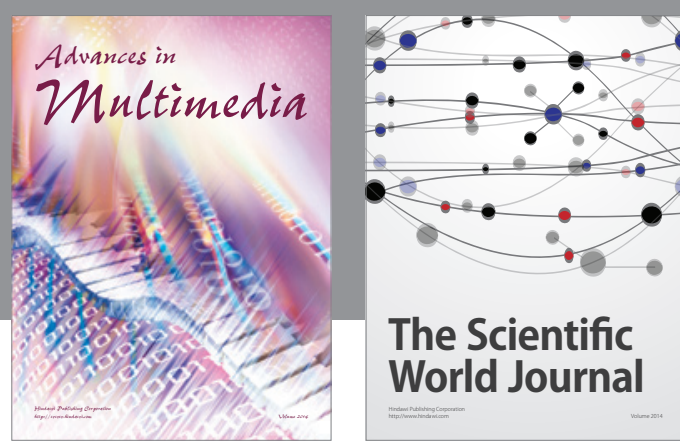

The Scientific World Journal
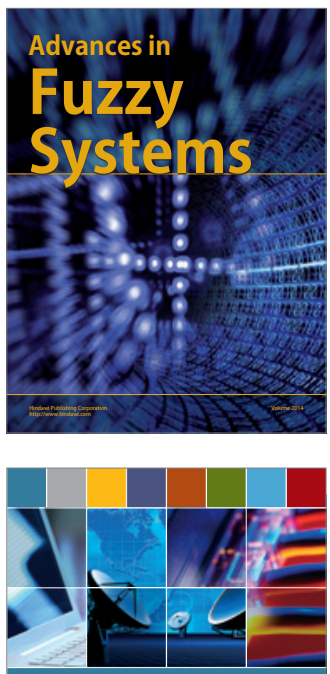

Computer Networks and Communications
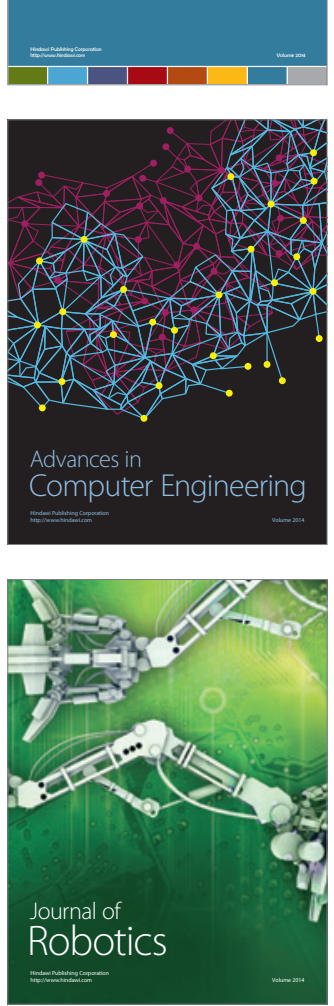
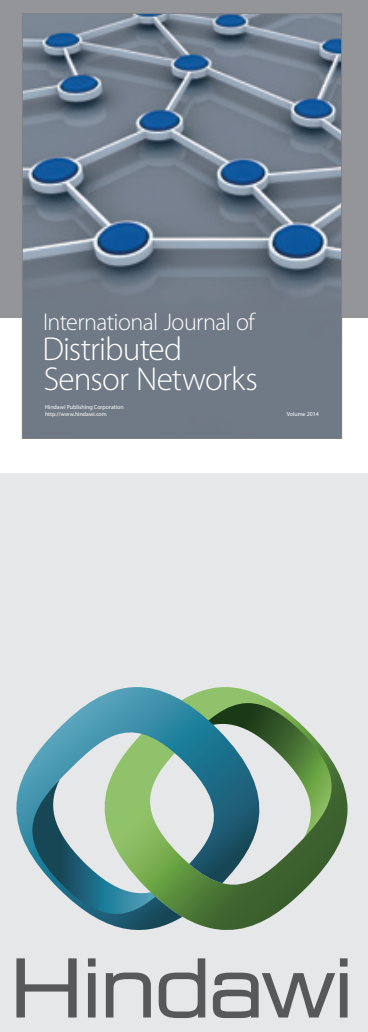

Submit your manuscripts at

http://www.hindawi.com
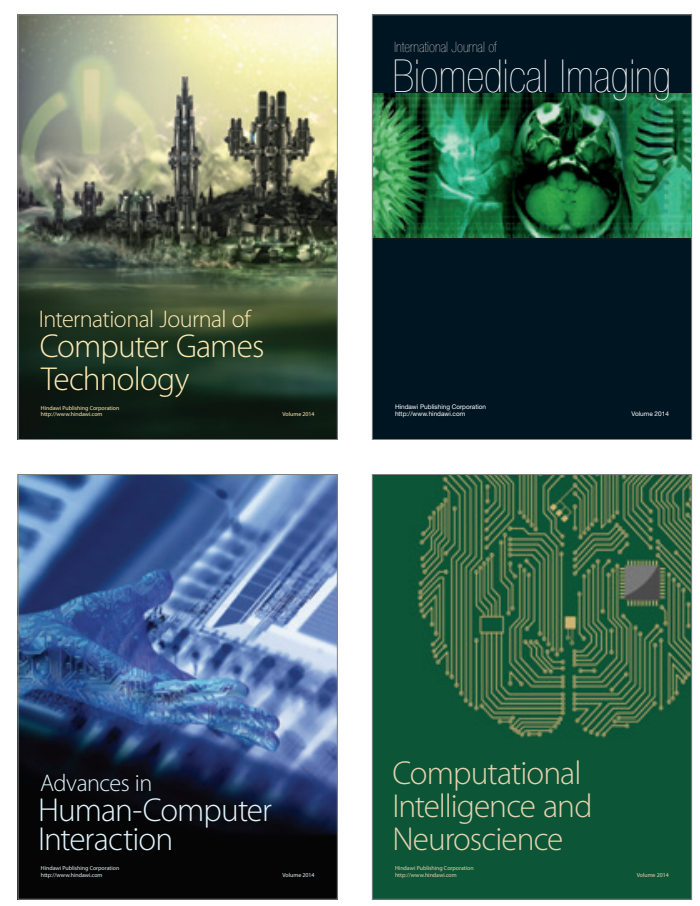
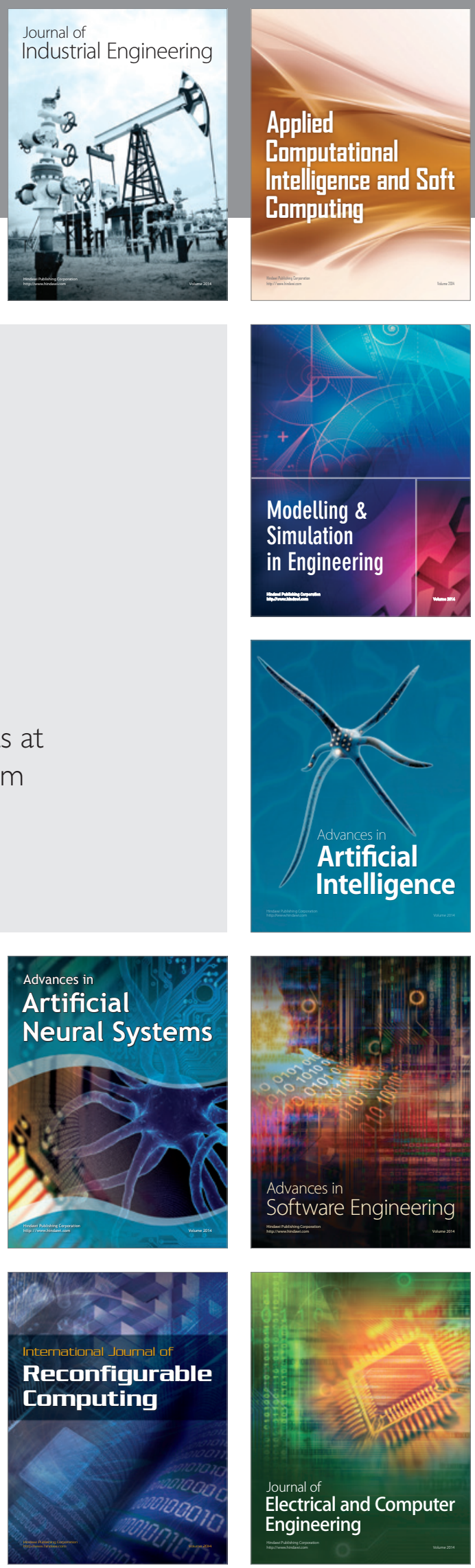